\title{
Graded doped structure fabricated by vacuum spray method to improve the luminance of polymer light -emitting diodes
}

\author{
X. Mo1, T. Mizokuro2,, ${ }^{*}$ C. Heck ${ }^{1}$, N. Tanigaki ${ }^{1}$ and T. Hiraga ${ }^{1}$
}

An increase in luminance of a polymer light-emitting diode (PLED) was obtained by fabricating a graded doping structure using a vacuum spray method. The small electron transport molecule, Tris(8-hydroxyquinolinato) aluminum(III)(Alq $\left.{ }_{3}\right)$, was graded dispersed along the film in the direction of growth in the hole transport polymer poly(3-hexylthiophene-2,5-diyl) (P3HT, regiorandom) layer of the PLED, despite being dissolved in the same organic solvent as the polymer. The PLED reported here, which is composed of a graded structure, emitted brighter light than PLEDs composed of pure polymer or of a blend of active layers prepared by spin coating and/or vacuum spray methods.

Keywords: Polymer light-emitting diodes; Luminance; Graded doped; Vacuum spray

Citation: X. Mo, T. Mizokuro, C. Heck, N. Tanigaki and T. Hiraga, "Graded doped structure fabricated by vacuum spray method to improve the luminance of polymer light-emitting diodes", Nano-Micro Lett. 1, 19-22 (2009). doi: 10.5101/nml.v1i1.p19-22

Organic and polymer light-emitting diodes (OLEDs and PLEDs

[1], respectively) have attracted a great deal of interest for application in emissive flat panel displays and as new light sources. One reason is that the OLEDs and PLEDs are composed by several layers on the substrate; thickness of these layers is less than $200 \mathrm{~nm}$. Therefore, these devices are lightweight and thin, and can be fabricated on flexible substrate. Now many efforts have been made to improve their luminance and lifetime. It has been shown, for example, that the luminance of an OLED can be improved by producing a bilayer (or multilayer) structure by successive sublimation steps. However, for PLEDs, it is usually difficult to fabricate a bi- [2] or multilayer [3] structure with common processes due to the dissolution between polymer layers.

Good balance between electron and hole injection is very important for the functioning of both OLEDs and PLEDs. In PLEDs, the most common way to achieve this balance is by blending the electron and hole transporting materials. The electroluminescent (EL) efficiency is higher when the electron and hole transport materials are separated and attached to the cathode and anode layers, respectively. Another way to achieve this balance could be by producing a structure in which the two materials are graded along the film direction of growth. As there is no interface between the electron and hole transport materials in this structure, it is expected that there will be an increase in the lifetime of the devices. For small organic molecules, graded structures can be fabricated by codeposition processes [4-7] and annealing [8], but for polymers it is difficult to control the gradient profile in the active layer due to dissolution by the solvent. There have been attempts to achieve polymer graded structures, such as molecular-scale interface engineering [9], self-organization [10], and thermal transfer process [11]; however, the processes involved are not simple.

We have developed a vacuum spray (VS) method [12-16]

${ }^{1}$ Materials Science Department, Fudan University, 220 Handan Road, Shanghai 200433, China

2 Photonics Research Institute, National Institute of Advanced Industrial Science and Technology, 1-8-31 Midorigaoka, Ikeda, Osaka 563-8577, Japan

*Corresponding author. Email: chem42@ni.aist.go.jp 


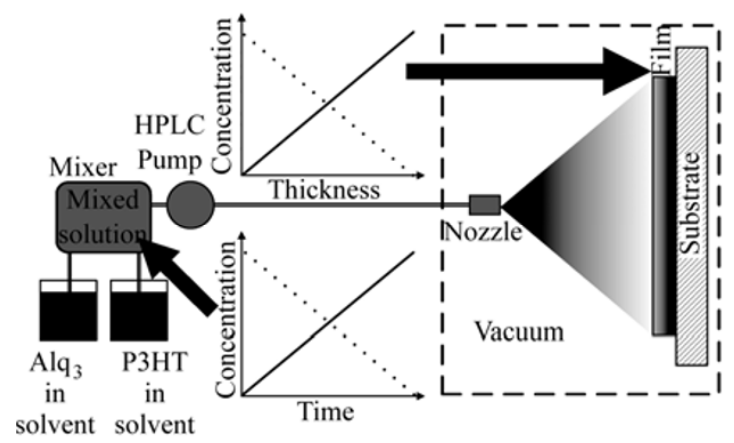

FIG. 1. Schematic illustration of the fabrication of polymer films with graded structure prepared by the VS method. The solid and dotted lines show the concentrations of $\mathrm{Alq}_{3}$ and P3HT, respectively.

that allows fabrication of polymer films with a controllable dye distribution along the film direction of growth, by which graded and/or bilayer structures can be prepared. This report describes the production of PLEDs based on films with homogenous and graded structures prepared using this VS method. For reference studies, devices based on spin-coated films were also investigated.

Figure 1 shows a schematic illustration of the preparation of a graded structured polymer film by the VS method. The polymer and small molecules are diluted separately in the same solvent. These two solutions are then mixed by an apparatus that is part of a gradient highperformance liquid chromatography (HPLC) pump, which includes a program that calculates and controls the precise output ratio of these solutions. This mixed solution is sprayed into a vacuum chamber through a pinhole nozzle, generating a solution mist that is then deposited on a rotating substrate. In the film preparation by VS method in this work, the vacuum chamber was evacuated below $5 \times 10^{-6} \mathrm{~Pa}$ by a turbo molecular pump, during the spray process, the chamber was maintained below $1 \times 10^{-1} \mathrm{~Pa}$. The spray flow rate was set at $1.7 \mathrm{ml} / \mathrm{min}$ by HPLC pump, the high spray pressure (from 35 to $40 \mathrm{MPa}$ ) was achieved. In addition, the substrate is heated with halogen lamps and the temperature $\left(\mathrm{T}_{\mathrm{s}}\right)$ can be precisely controlled. In this work, $\mathrm{T}_{\mathrm{s}}$ was controlled at $353 \mathrm{~K}$. Substrate heating combined with the low pressure in the chamber leads to rapid and complete evaporation of the solution when the mist reaches the substrate. As polymer and small molecules hardly migrate without solvent on the substrate, the concentration distribution of small molecules in the polymer matrix, in the film direction of growth, is determined by their arrival ratio. Films with homogeneous structures were prepared by spraying a homogenously blended solution into the chamber. To prepare a graded polymer film containing increasing concentrations of small molecules along the film direction of growth, the

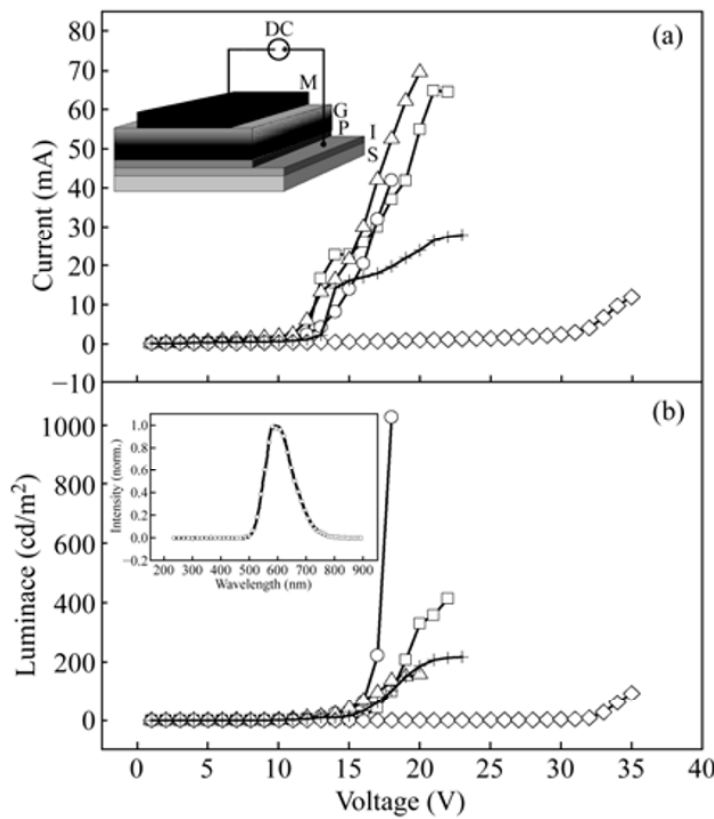

$\triangle$ Pure P3HT, spin coating $\quad \longrightarrow-$ Pure P3HT, spray

$\multimap$ P3HT: Alq3 blend, spin coating — P3HT: Alq3 blend, spray

$\multimap-$ P3HT: Alq3 graded, spray

FIG. 2. (a) $I-V$, (b) $L-V$ characteristics of PLEDs with active layers prepared by spin coating and VS methods. The inset in (a) shows a PLED with a graded structure. S, substrate; I, ITO; P, PEDOT:PSS; G, Polymer layer with graded structure; $\mathrm{M}, \mathrm{Mg}: \mathrm{Ag}$. The inset in (b) shows the EL spectrum of a PLED with a graded structure.

concentration of small molecules was increased linearly relative to the polymer concentration during the deposition process. The thickness of the film is controlled by the solution concentration, flow rate, and spraying time.

PLEDs were produced by spin-coating a hole-injecting material, i.e., poly (3,4-ethylenedioxythiophene) poly (styrenesulfonate) (PEDOT:PSS; Aldrich), on the indium-tin- oxide substrate. An active layer $110 \mathrm{~nm}$ thick was then produced by the VS method where the Tris(8-hydroxyquino-linato) aluminum(III) ( $\mathrm{Alq}_{3}$; Aldrich) electron-transporting molecules were dispersed in the poly (3-hexylthiophene-2, 5-diyl) (P3HT) (regiorandom; Aldrich) hole-transporting polymer. Finally, a $\mathrm{Mg}: \mathrm{Ag}$ (10:1) cathode $50 \mathrm{~nm}$ thick was deposited on top by thermal evaporation. Each PLED cell was $2 \mathrm{~mm} \times 2 \mathrm{~mm}$ in size.

Three types of active layer were prepared by the VS method with substrate temperature fixed at $353 \mathrm{~K}$. Pure P3HT layers were prepared with $\mathrm{P} 3 \mathrm{HT} /$ chloroform solution at concentration of $0.06 \mathrm{wt} \%$; blend P3HT:Alq ${ }_{3}$ (4:1) layers with P3HT:Alq 3 (4:1)/chloroform solution, in which P3HT concentration was $0.048 \mathrm{wt} \%$ and $\mathrm{Alq}_{3}$ concentration was 0.012 $\mathrm{wt} \%$; and graded layers of P3HT and $\mathrm{Alq}_{3}$ with P3HT/ chloroform and $\mathrm{Alq}_{3} /$ chloroform solutions both at concentration of $0.06 \mathrm{wt} \%$. During the production of the graded layers, the 
$\mathrm{Alq}_{3}: \mathrm{P} 3 \mathrm{HT}$ ratio was increased linearly. For reference studies, P3HT and P3HT:Alq $\mathrm{Al}_{3}$ blend films were prepared by spin coating. The P3HT spin coating film was prepared with P3HT/chloroform solution at the concentration of $1.0 \mathrm{wt} \%$, The P3HT:Alq ${ }_{3}$ blend spin coating film was prepared with blend solution in which the concentration of P3HT was $0.8 \mathrm{wt} \%$ and the concentration of $\mathrm{Alq}_{3}$ was $0.2 \mathrm{wt} \%$.

Figure 2 shows the electroluminescence properties of the PLEDs prepared by spin coating and VS methods. While the $I-V$ characteristics (Fig. 2a) did not differ with production method for PLEDs composed of a pure P3HT layer, the $L-V$ characteristics showed that PLEDs prepared by the VS method emitted brighter light. This may have been because there is less solvent residue in the polymer layer prepared by the VS method.

Adding an electron transport material to the hole transport material is one way to achieve better electron injection and to balance the carriers, and thus higher luminance can be expected. In this study, the electron transport material $\left(\mathrm{Alq}_{3}\right)$ was added into the hole transport (P3HT) material and the results are shown in Fig. 2. In the case of PLEDs with uniformly blended layers, both the current and the luminance of PLEDs prepared with the spray layer were higher than those of PLEDs prepared with the spin-coated layer. This may be because solvent residue is left in the spin-coated layer and/or that $\mathrm{Alq}_{3}$ aggregates easily in layers prepared by the spin coating process due to the solvent residue. With the VS method, where almost no solvent residue is left in the layer, $\mathrm{Alq}_{3}$ molecules did not migrate after reaching the substrate, which markedly impeded the aggregation.

As a general trend, PLEDs with a blend structure have higher working voltage [17], and in this study the turn-on voltage $\left(V_{o n}\right.$, at the minimum luminance of $\left.0.1 \mathrm{~cd} / \mathrm{m}^{2}\right)$ of the PLEDs with a spin coating layer (see Table 1) confirmed this trend. However, the $V_{\text {on }}$ of the PLEDs with a blend layer prepared by VS method was about the same as that of PLEDs composed of pure structures. This low $V_{\text {on }}$ is an advantage of the VS method for PLED applications. Unfortunately, the highest measured luminance $\left(L_{\max }\right)$ of the PLEDs with a blend layer was lower than the $L_{\max }$ of PLEDs with pure structures. In the blend structure case, the efficiency was even lower than that of the PLEDs with a pure P3HT active layer.

To improve the luminance of PLEDs, the blended layer was exchanged for a graded doped layer where the structure consisted of a P3HT matrix in which the concentration of $\mathrm{Alq}_{3}$ was increased linearly from 0 to $20 \%$. Thus, while there is no $\mathrm{Alq}_{3}$ in the polymer near the interface with the PEDOT:PSS layer, the concentration of $\mathrm{Alq}_{3}$ increases linearly in the polymer until about $20 \%$ in the region near the interface with the cathode. We have reported the structure of these graded layers prepared by VS method analyzed by scanning transmission electron microscopy (STEM) $[14,15]$. Although in the present study the polymer film was thinner, the graded structure is expected to be similar to that reported previously.

The graded structure device has a turn-on voltage of $9 \mathrm{~V}$, which is about the same as that of the pure and blend structure devices (Tab. 1), however, it emits much brighter light than the pure and graded structure devices (Fig. 2b). As the heterojunction interfaces are virtually eliminated in the graded structure, this increase in brightness was probably because there is a better balance of charge carrier in such a way that there is a higher recombination probability of holes and electrons [5], leading to a large increase in PLED performance. In the present study, the optimized highest ratio of $\mathrm{Alq}_{3}$ to P3HT was about $1: 4$, but suitable ratios have still to be determined for other combinations of electron and hole transport materials.

All PLEDs showed very similar EL spectra to that of the graded structured PLED shown in the insert of Fig. 2b. In addition, all PLEDs emitted yellow-orange light and the preparation method and structure of active layers did not influence the color. Thus, only P3HT emit light, while $\mathrm{Alq}_{3}$ acted only as an electron transport material without emitting light.

In summary, this report described the successful fabrication of a PLED in which the polymer active layer had graded doping with electron transport molecules by the VS

\begin{tabular}{|c|c|c|c|c|}
\hline \multirow{2}{*}{ Device Structure } & \multicolumn{2}{|c|}{ Spin coating } & \multicolumn{2}{|c|}{ VS method } \\
\hline & $V_{o n}(\mathrm{~V})$ & $L_{\max }\left(\mathrm{cd} / \mathrm{m}^{2}\right)$ & $V_{o n}(\mathrm{~V})$ & $L_{\max }\left(\mathrm{cd} / \mathrm{m}^{2}\right)$ \\
\hline Pure P3HT & 7 & 159.4 & 9 & 415.1 \\
\hline $\begin{array}{l}\text { P3HT:Alq } \\
\text { (4:1) blend }\end{array}$ & 23 & 94 & 9 & 218.8 \\
\hline $\begin{array}{c}\text { P3HT:Alq } \\
\text { Graded }\end{array}$ & - & - & 9 & 1028.0 \\
\hline
\end{tabular}


method. The graded doped PLED emitted much brighter light than those produced with a pure polymer layer and with a blend layer, regardless of whether the layers were prepared by the spin coating or spray method. Due to the excellent controllability of the doping process of the VS method, other structures such as bilayer, multilayer, or more complicated profiles can also be prepared. The conversion efficiency of not only PLEDs but also polymer solar cells can be improved by using a graded structure. Therefore, the VS method is very promising for the fabrication of polymer optoelectronic devices, not only PLEDs but also polymer solar cells.

This work was supported by the Industrial Technology Research Grant Program from the New Energy and Industrial Technology Development Organization (NEDO) of Japan. The authors would like to thank Technical Support Co, Ltd. Japan for manufacturing the vacuum spray apparatus.

Received 24 September 2009; accepted 5 October 2009; published online 15 October 2009.

\section{References}

1. J. H. Burroughes, D. D. C. Bradley, A. R. Brown, R. N. Marks, K. Mackay, R. H. Friend, P. L. Burns and A. B. Holmes, Nature (London) 347, 539 (1990). doi:10.1038/ $\underline{347539 \mathrm{a} 0}$

2. Y. Xia and R. H. Friend, Appl. Phys. Lett. 88, 163508 (2006). doi:10.1063/1.2196229

3. S. R. Tseng, S. C. Lin, H. F. Meng, H. H. Liao, C. H. Yeh, H. C. Lai, S. F. Horng and C. S. Hsu, Appl. Phys. Lett. 88, 163501 (2006). doi:10.1063/1.2192574

4. Y. Shao and Y, Yang, Appl. Phys. Lett. 83, 2453 (2003). doi:10.1063/1.1605800

5. D. Ma, C. S. Lee, S. T. Lee and L. S. Hung, Appl. Phys. Lett. 80, 3641 (2002). doi:10.1063/1.1479450

6. B. D. Chin, M. C. Suh, M. -H. Kim, S. T. Lee, H. D. Kim and H. K. Chung, Appl. Phys. Lett. 86, 133505 (2005). doi:10.1063/1.1894596

7. A. B. Chwang, R. C. Kwong and J. J. Brown, Appl. Phys. Lett. 80, 725 (2002). doi:10.1063/1.1446992

8. C. W. Chen, T. Y. Cho, C. C. Wu, H. L. Yu and T. Y. Luh, Appl. Phys. Lett. 81, 1570 (2002). doi:10.1063/ $\underline{1.1502912}$

9. P. K. H. Ho, H. -S. Kim, J. H. Burroughes, H. Becker, S. F. Y. Li, T. M. Brown, F. Cacialli and R. H. Friend, Nature (London) 404, 481 (2000). doi:10.1038/35006610

10. T. -W. Lee, Y. Chung, O. Kwon and J. -J. Park, Adv, Funct. Mater. 17, 390 (2007). doi:10.1002/adfm.200600278

11. H. H. Chang, C. C. Wu, C. C. Yang, C. W. Chen and C. C. Lee, Appl. Phys. Lett. 78, 574 (2001). doi:10.1063/ 1.1344223

12. T. Hiraga, N. Tanaka, S. Takarada, K. Hayamizu, N. Kikuchi and T. Moriya, J. Vac. Sci. Technology A 12, 876 (1994). doi:10.1116/1.579270

13. X. L. Mo, T. Mizokuro, H. Mochizuki, N. Tanigaki and T. Hiraga, Jpn. J. Appl. Phys. 43, 307 (2004). doi:10.1143/ JJAP.43.307

14. X. L. Mo, T. Mizokuro, A. Kobayashi, G. R. Chen, N. Tanigaki and T. Hiraga, Thin Solid Films 516, 1663 (2008). doi:10.1016/j.tsf.2007.05.013

15. X. L. Mo, T. Mizokuro, A. Kobayashi, G.R. Chen, H. Mochizuki, N. Tanigaki and T. Hiraga, Jpn. J. Appl. Phys. 45, 231 (2006). doi:10.1143/JJAP.45.231

16. X. L. Mo, T. Mizokuro, N. Tanigaki, T. Hiraga, N. Umehara, K. Takagi and S. Yamamoto, Jpn. J. Appl. Phys. 47, 425 (2008). doi:10.1143/JJAP.47.425

17. M. Uchida, C. Adachi, T. Koyama and Y. Taniguchi, J. Appl. Phys. 86, 1680 (1999). doi:10.1063/1.370947 